\title{
MyD88 Is Required for Efficient Control of Coxiella burnetii Infection and Dissemination
}

\author{
Lisa Kohl ${ }^{1}$, Inaya Hayek ${ }^{1}$, Christoph Daniel ${ }^{2}$, Jan Schulze-Lührmann ${ }^{1}$, \\ Barbara Bodendorfer ${ }^{1}$, Anja Lührmann ${ }^{1}$ and Roland Lang ${ }^{1 *}$ \\ ${ }^{1}$ Institute of Clinical Microbiology, Immunology and Hygiene, Universitätsklinikum Erlangen, Friedrich-Alexander-Universität \\ Erlangen-Nürnberg, Erlangen, Germany, ${ }^{2}$ Department of Nephropathology, Institute of Pathology, Universitätsklinikum \\ Erlangen, Friedrich-Alexander-Universität Erlangen-Nürnberg, Erlangen, Germany
}

\section{OPEN ACCESS}

Edited by:

Jason Paul Gigley, University of Wyoming, United States

Reviewed by:

Sunny Shin,

University of Pennsylvania,

United States

Catherine Ropert,

Universidade Federal de Minas

Gerais, Brazil

*Correspondence:

Roland Lang

roland.lang@uk-erlangen.de

Specialty section: This article was submitted to

Microbial Immunology,

a section of the journal

Frontiers in Immunology

Received: 15 June 2018 Accepted: 18 January 2019 Published: 08 February 2019

Citation:

Kohl L, Hayek I, Daniel C, Schulze-Lührmann J, Bodendorfer B,

Lührmann A and Lang R (2019) MyD88 Is Required for Efficient Control of Coxiella burnetii Infection and Dissemination.

Front. Immunol. 10:165. doi: 10.3389/fimmu.2019.00165
The intracellular pathogen Coxiella (C.) burnetii causes $Q$ fever, a usually self-limiting respiratory infection that becomes chronic and severe in some patients. Innate immune recognition of $C$. burnetii and its role in the decision between resolution and chronicity is not understood well. However, TLR2 is important for the response to C. burnetii in mice, and genetic polymorphisms in Myd88 have been associated with chronic $Q$ fever in humans. Here, we have employed MyD88-deficient mice in infection models with the attenuated C. burnetii Nine Mile phase II strain (NMII). Myd88-/- macrophages failed to restrict the growth of NMII in vitro, and to upregulate production of the cytokines TNF, IL-6, and IL-10. Following intraperitoneal infection, NMII bacterial burden was significantly higher on day 5 and 20 in organs of $M y d 88^{-/-}$mice. After infection via the natural route by intratracheal injection, a higher bacterial load in the lung and increased dissemination of NMII to other organs was observed in MyD88-deficient mice. While wild-type mice essentially cleared NMII on day 27 after intratracheal infection, it was still readily detectable on day 42 in multiple organs in the absence of MyD88. Despite the elevated bacterial load, Myd88-/- mice had less granulomatous inflammation and expressed significantly lower levels of chemoattractants, inflammatory cytokines, and of several IFN $\gamma$-induced genes relevant for control of intracellular pathogens. Together, our results show that MyD88-dependent signaling is essential for early control of $C$. burnetii replication and to prevent systemic spreading. The continued presence of NMII in the organs of $\mathrm{Myd} \mathrm{S}^{-1-}$ mice constitutes a new mouse model to study determinants of chronicity and resolution in $Q$ fever.

Keywords: Coxiella burnetii nine mile phase II, Toll-Like Receptor (TLR), Q fever, mouse model, chronicity, resolution, intratracheal

\section{INTRODUCTION}

The intracellular bacterium Coxiella burnetii is the pathogenic agent of the zoonotic disease $\mathrm{Q}$ fever, which usually presents as self-limiting respiratory tract infection after inhalation of aerosolized bacteria shed by infected small ruminants. In a small percentage of human patients, C. burnetii infection does not resolve, but develops into severe and chronic infection affecting the vasculature, including endocarditis. Host factors associated with an increased risk to develop chronic Q fever are older age, cardiac valve abnormalities, pregnancy and immunosuppression $(1,2)$. 
Key immunological host factors required to control C. burnetii infection have been identified in the mouse model. Since C. burnetii resides and proliferates intracellularly, mostly in macrophages, it is not surprising that protective host immunity appears to rely on $\mathrm{T}$ cells and IFN $\gamma$, as is the case in other intracellular infections. The pivotal importance of $\mathrm{T}$ cells has been demonstrated in SCID mice and nude mice (3). Production of IFN $\gamma$, produced by Th1, CD8 ${ }^{+} \mathrm{T}$ cells or NK cells, is essential to control infection with C. burnetii in the murine system (3). By which mechanisms IFN $\gamma$ signaling induces the killing of C. burnetii in macrophages is only incompletely understood, but involves the production of reactive nitrogen intermediates by iNOS, at least in the murine model (4). Interestingly, the production of IFN $\gamma$ appears not to be deficient in patients with chronic Q fever (5), suggesting that other host factors are involved. The immunomodulatory cytokine IL-10 deactivates macrophages through Stat3-dependent signaling, leading to impaired production of cytokines like TNF and IL-12 (6). IL-10 is overproduced by monocytes of patients with chronic Q fever (7) and impairs killing of C. burnetii in human macrophages (8). In addition, mice overproducing IL-10 from macrophages (9) have higher and prolonged bacterial burden after infection with $C$. burnetii (10), constituting a mouse model for chronic $Q$ fever.

Impaired sensing of C. burnetii by the innate immune system may be another explanation for the development of chronic infection in some patients. This notion is in fact supported by the demonstration that a single nucleotide polymorphism in the Toll-like receptor (TLR) adapter protein MyD88 was associated with development of chronic $\mathrm{Q}$ fever in a large cohort of Dutch patients (11). A role for TLR2 as pattern recognition receptor for C. burnetii was already established in 2004 in mouse macrophages (12) and has been confirmed in human cells (13). MyD88 was recently demonstrated to be required for induction of TNF production and control of bacterial replication in murine macrophages infected with C. burnetii in vitro (14). Furthermore, TLR2- and MyD88-deficient mice developed increased bacterial burden after intratracheal infection with C. burnetii (15).

Coxiella burnetii shows phase variation with regard to LPS synthesis. Phase I C. burnetii synthesizes LPS with a highly branched O-chain, which has been traditionally considered the major virulence factor because it is the form isolated from patients with Q fever (16). Serial in vitro culture resulted in a shift to phase II LPS variants with truncated O-antigen polysaccharides, which in the case of the C. burnetii Nine Mile phase II clonal derivative is due to a chromosomal $26 \mathrm{kB}$ deletion affecting several LPS biosynthesis genes (17). Since the C. burnetii Nine Mile Phase 2 RSA 439 clone 4 (NMII) was found to be less virulent than the phase I parent strain in immunocompetent mice and guinea pigs $(18,19)$, it can be used under Biosafety Level 2 conditions. Importantly, both phase variants show similar growth in a modified phagosome, the Coxiella-containing vacuole of murine and human macrophages $(20,21)$. Furthermore, recent studies have clearly shown that the attenuated NMII strain is virulent in SCID mice that lack T and B lymphocytes $(22,23)$,

Abbreviations: TLR, Toll-like receptor; NMII, C. burnetii Nine Mile phase II; GE, Genome equivalents. demonstrating that virulence of $C$. burnetii cannot be reduced to phase I LPS, and providing an opportunity to study bacterial and host factors that determine the course of infection in a more amenable mouse model.

Here, we have used MyD88-deficient mice to investigate the course of infection with the attenuated NMII strain. We found that MyD88 was required in macrophages in vitro for restricting growth of NMII and for expression and secretion of cytokines. Myd88-/- mice developed higher bacterial loads in spleen, liver and lung after intraperitoneal infection. This phenotype was confirmed after intratracheal infection with NMII, where an essential role of MyD88 in efficient elimination of NMII from the lung and from other tissues, including the heart, was observed. In the absence of MyD88, mice had reduced granulomatous inflammation and showed impaired expression of proinflammatory cytokines and chemokines, as well as a reduced IFN $\gamma$-response. Infection of $M y d 88^{-/-}$mice with the attenuated NMII strain proved to model important aspects of chronic Q fever (prolonged C. burnetii load in several tissues; impaired granuloma formation; and altered cytokine expression). We therefore propose this experimental setting as a promising model to further explore the contribution of host factors, such as IFN $\gamma$-induced genes, but also of bacterial factors, to the control of infection and resolution of inflammation.

\section{RESULTS}

\section{NMII Replicates in MyD88-Deficient BMM}

We first tested the role of MyD88 signaling in the interaction of C. burnetii Nine Mile phase II (NMII) with macrophages in vitro. Bone marrow-derived macrophages (BMM) from $M y d 88^{-/-}$ and $M y d 88^{+/-}$or C57BL/6 control mice were infected and analyzed over time for the growth or persistence of NMII by qPCR for the bacterial $\operatorname{dotA}$ gene and the murine albumin gene (Figure 1A) and immunofluorescence detection of NMII (Figure 1B). Early after infection, at $4 \mathrm{~h}$, comparable levels of NMII genome equivalents (GE) per macrophage were found by qPCR, indicating that MyD88 is not controlling the uptake or early killing of NMII. While wild type macrophages controlled NMII, with moderately decreasing signals detected by qPCR and by immunofluorescence after 24 and $72 \mathrm{~h}$, the NMII GE number per macrophage already increased after $24 \mathrm{~h}$ and continued to do so after $72 \mathrm{~h}$, reaching a roughly five-fold higher level compared to the $4 \mathrm{~h}$ time point. Immunofluorescence staining of NMII revealed the development of large Coxiella-containing vacuoles $(\mathrm{CCV})$ in a quite large fraction of $M y d 88^{-/-}$BMM after $72 \mathrm{~h}$, whereas the size of the $\mathrm{CCV}$ did not augment in wild type BMM (Figure 1B). Thus, MyD88 was not involved in the uptake of NMII, but was essential to inhibit bacterial replication and development of a large CCV.

\section{Differential Requirement for MyD88 in Cytokine Production and Enzyme Expression by NMII-infected Macrophages}

The production of TNF, IL- 6 , and IL-10 by BMM infected with NMII was robustly detectable in supernatants of wildtype 
A

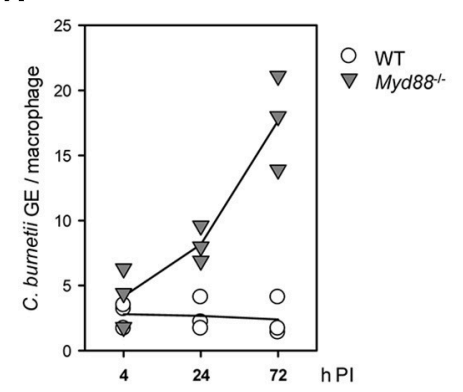

B

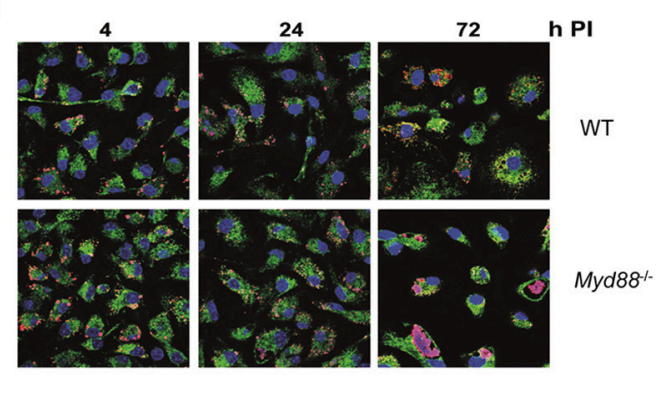

FIGURE 1 | Replication of NMII in MyD88-deficient macrophages. MyD88-deficient (Myd88-/-) and wild-type BMM (WT) were infected with NMII (MOI of 10). (A) At the indicated time points post-infection (PI), bacterial load was assessed by qPCR for the C. burnetii dotA gene and the murine albumin gene. Results of three independent experiments are shown. (B) BMM were infected on glass coverslips. The cells were fixed and stained at the indicated time points with DAPI (blue) and with antibodies against LAMP1 (green) and C. burnetii (red). One of 3 experiments with similar results is shown.

BMM and completely dependent on MyD88 (Figure 2A). Measurement of mRNA induction by qRT-PCR confirmed this effect and dependence on MyD88 for IL-6 and IL-10 (Figure 2B). Of interest, induced expression of INOS, a critical anti-microbial enzyme involved in control of C. burnetii $(4,24)$, was induced to the same level in BMM from $M y d 88^{-/-}$as wildtype mice. Upregulation of the other arginine-utilizing enzyme in macrophages, Arginase-1, by NMII was again completely abrogated in $M y d 88^{-/-}$BMM. Expression of $I l 12 b$ mRNA was strongly and MyD88-dependently induced by NMII after 2-6 h, and down-regulated after $24 \mathrm{~h}$ (Figure 2C). Together, these data identified a critical role for MyD88 in the induction of important pro- and anti-inflammatory cytokines in response to NMII infection in vitro, but also suggest that not all transcriptional responses in NMII-infected BMM require MyD88 signaling.

\section{Increased NMII Burden in Spleen, Liver and Lung After Intraperitoneal Infection}

To determine whether MyD88-dependent signaling is required for control of NMII in vivo, mice were first infected by the intraperitoneal route with $5 \times 10^{7}$ NMII. A moderate but significant decrease in body weight was observed in $M y d 88^{+/-}$ mice on day 2 after infection, but not in $M y d 88^{-/-}$mice (Figure 3). However, both $M y d 88^{+/-}$and $M y d 88^{-/-}$mice did not show signs of disease, appeared normal following infection and gained weight at a similar rate after 4 days of infection. The bacterial burden in spleen, liver and lung was analyzed by qPCR detection of the C. burnetii insertion sequence IS1111 as genome equivalents (GE) per cell on day 5 and 20 after i.p. infection (Figure 4A). NMII GE on day 5 were comparable between genotypes in the spleen but significantly higher in liver and lung of $M y d 88^{-/-}$mice. On day 20, the NMII load was already reduced by 4 orders of magnitude compared to day 5 levels in $M y d 88^{+/-}$organs, indicating efficient and ongoing elimination of bacteria. In contrast, bacterial load decreased less efficiently in $M y d 88^{-/-}$organs, where the NMII GE were significantly higher than in $M y d 88^{+/-}$spleen, liver and lung. To complement quantitative PCR measurement of NMII genomes by an independent method showing the spatial distribution of bacteria in the tissue, we employed immunohistochemical detection of NMII in liver tissue at both time points (Figures 4B,C).

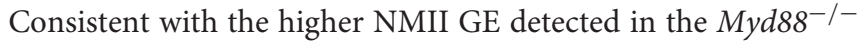
liver already at day 5, we observed more Coxiella-positive spots in the $M y d 88^{-/-}$liver sections. This difference was again more pronounced at day 20, when most liver sections of $M y d 88^{+/-}$ mice were negative for immunohistochemical staining of $C$. burnetii, but $M y d 88^{-/}$livers still contained easily detectable bacteria. Thus, both detection of NMII GE by qPCR and immunohistochemical staining of NMII consistently showed significantly higher bacterial burden in $M y d 88^{-/-}$organs after intraperitoneal infection, which was more pronounced at the later time point (day 20) after infection. Nevertheless, also $M y d 88^{-/-}$mice could reduce bacterial burden between day 5 and 20, albeit less efficiently than $M y d 88^{+/-}$controls.

\section{Increased Dissemination and Persistence of NMII in Myd88-/- Mice After Intratracheal Infection}

The natural route of infection with C. burnetii is via the airways by aerosolized bacteria. Recently, a higher permissiveness of alveolar macrophages for C. burnetii replication has been reported for in vitro as well as in vivo infection $(25,26)$. Therefore, we infected mice by intratracheal injection with $10^{6}$ NMII and determined weight loss as sign of clinical disease and the bacterial load as GE by qPCR in different organs over time (Figure 5). Similar to what was observed after intraperitoneal infection, $M y d 88^{+/-}$and $M y d 88^{+/+}$mice experienced a transient and moderate loss in weight with a nadir at $\mathrm{d} 2$ post intratracheal infection, but regained weight within 1 week (Figure 5A). In contrast, MyD88-deficient mice were protected from weight loss after intratracheal infection. Both $M y d 88^{-/-}$and $M y d 88^{+/-}$mice did not show signs of clinical disease during the further course of the experiment ( $\mathrm{d} 7$ to $\mathrm{d} 120$ ). As expected, the highest NMII GE were found in the lung at the early time point on day 7 . While $M y d 88^{+/-}$mice efficiently reduced NMII in the lungs already at day 16 and eliminated the bacteria before day 42 , in $M y d 88^{-/-}$mice NMII GE were already higher on day 7 , and this difference between genotypes 

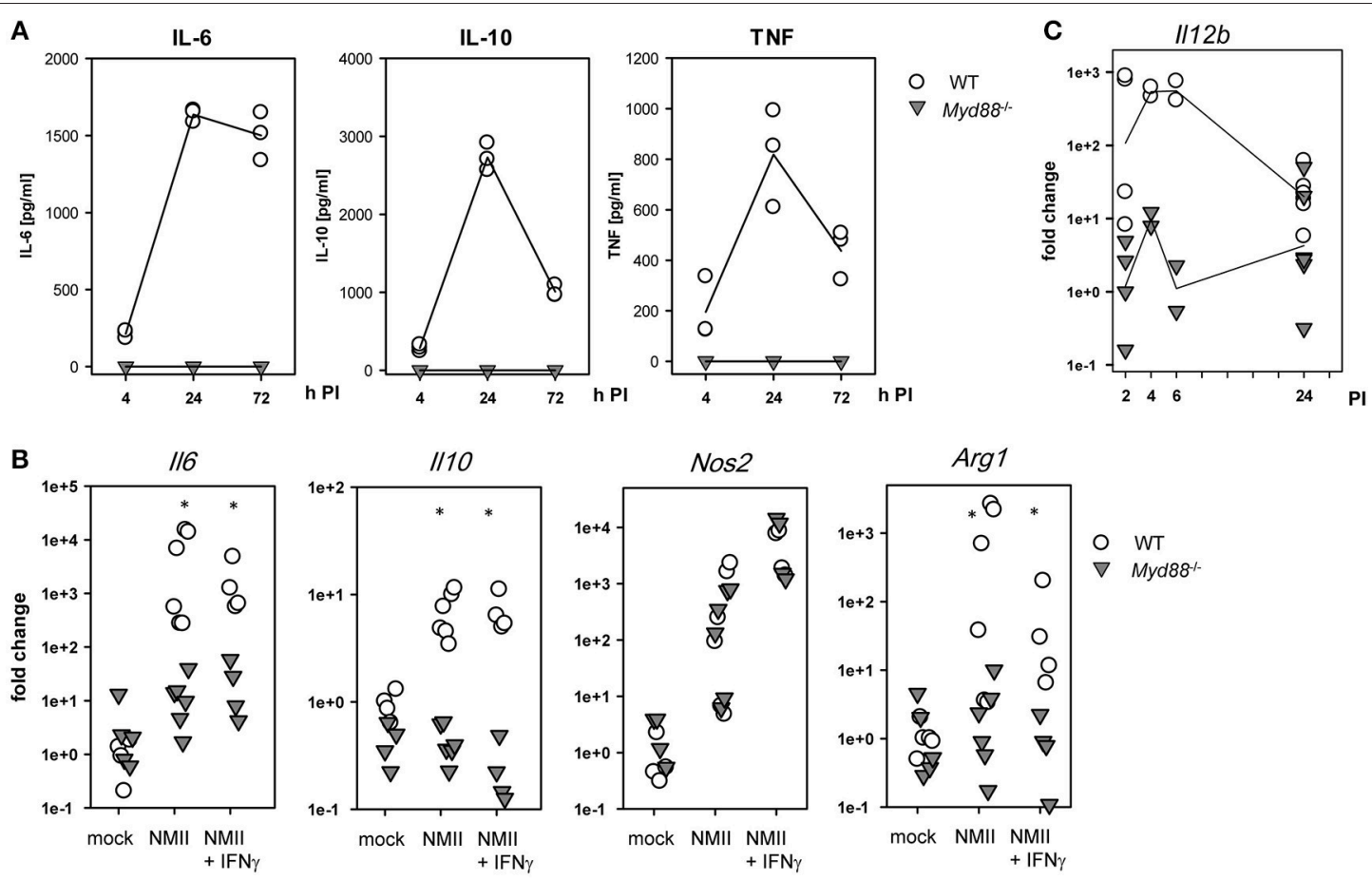

FIGURE 2 | Dependence of C. burnetii-induced cytokine and enzyme expression on MyD88 in BMM. (A) supernatants of BMM infected with NMII at an MOI of 10 were used to determine secreted cytokine levels by ELISA of IL-6, IL-10, and TNF. Mean values of duplicate wells from three independent experiments are shown. (B) BMM were plated in antibiotic-free cDMEM overnight. NMII was added to the BMM (MOI 10) in the presence or absence of IFN $\gamma$ (10 ng/ml). After $3 \mathrm{~h}$, extracellular NMII was washed away. Cells were lysed after $24 \mathrm{~h}$ for preparation of RNA. Relative quantitation was performed by $\Delta \Delta \mathrm{CT}$ method, using the mean $\Delta \mathrm{CT}$ of non-infected BMM as calibrator. Pooled data from two (NMII+IFN $\gamma$ ) and three experiments (mock / NMII) performed with biological duplicates are shown. Mann-Whitney Rank Sum Test, asterisks indicate $p<0.05$. (C) kinetic analysis of $/ 12 b$ mRNA expression. RNA was harvested form BMM at the indicated times after infection with NMII (MOI 10) and processed for qRT-PCR. Pooled data from 3 experiments, each dot represents RNA from a biological replicate.

increased on days 16,27 , and 42 . Even after 4 months, NMII was readily detectable by $\mathrm{qPCR}$ in the lungs of three out of four $M y d 88^{-/-}$mice, indicating long term pulmonary persistence. NMII spread systemically to all organs analyzed here and was detectable on day 7 after intratracheal infection by qPCR in decreasing quantity in liver, spleen and heart of $M y d 88^{+/-}$ (Figure 5). Of interest, $M y d 88^{-/-}$mice harbored a substantially higher bacterial load in spleen, liver and the heart. In fact, while NMII was almost completely cleared from these organs in Myd88 $8^{+/-}$mice already on day 27, it was still readily detectable on d42 in most $M y d 88^{-/-}$mice. However, in contrast to the continued persistence in $M y d 88^{-/-}$lungs after 4 months, at this late time point NMII appeared to be eliminated from spleen, liver, and heart, based on negative qPCR results. Together, the results from the intratracheal infection experiments corroborated the findings made after intraperitoneal infection, and showed that MyD88 signaling is essential for local control of NMII in the lung after intratracheal infection, preventing systemic dissemination and initiating clearance of NMII from organ tissues.

\section{Decreased Granulomatous Inflammation in Myd88-/- Mice After NMII Infection}

Despite the increased and prolonged presence of NMII in the organ tissues, $M y d 88^{-/-}$mice did not develop signs of

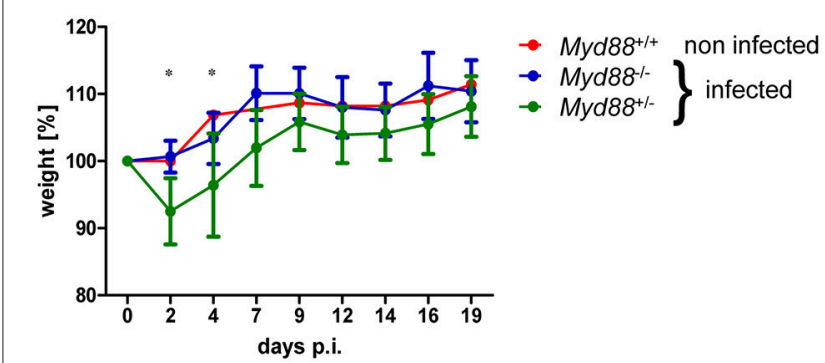

FIGURE 3 | Transient weight loss after NMII infection. Mice were weighed before and at indicated time points after intraperitoneal infection with $5 \times 10^{7}$ NMII. Changes in weight relative to day 0 are shown (mean $+/-$ SD). One non-infected Myd88 $+/+$ mouse was included for comparison. ( $n=8$ (day $0-5$ ) and $n=4$ (day 6-20) for Myd88 ${ }^{+/-}$), $n=7$ (day 0-5) and $n=3$ (d6-20) for $\mathrm{Myd} 88^{-/-}$). Results were analyzed using an unpaired two-tailed Mann-Whitney $\cup$ test.

clinical disease and in fact were protected from the transient weight loss induced early after infection in $M y d 88^{+/-}$mice (Figure 3). To investigate the impact of MyD88 signaling on leukocyte infiltration and tissue damage, we next performed histopathological analyses of livers obtained on day 5 and 20 after intraperitoneal infection (Figure 6). In the livers 

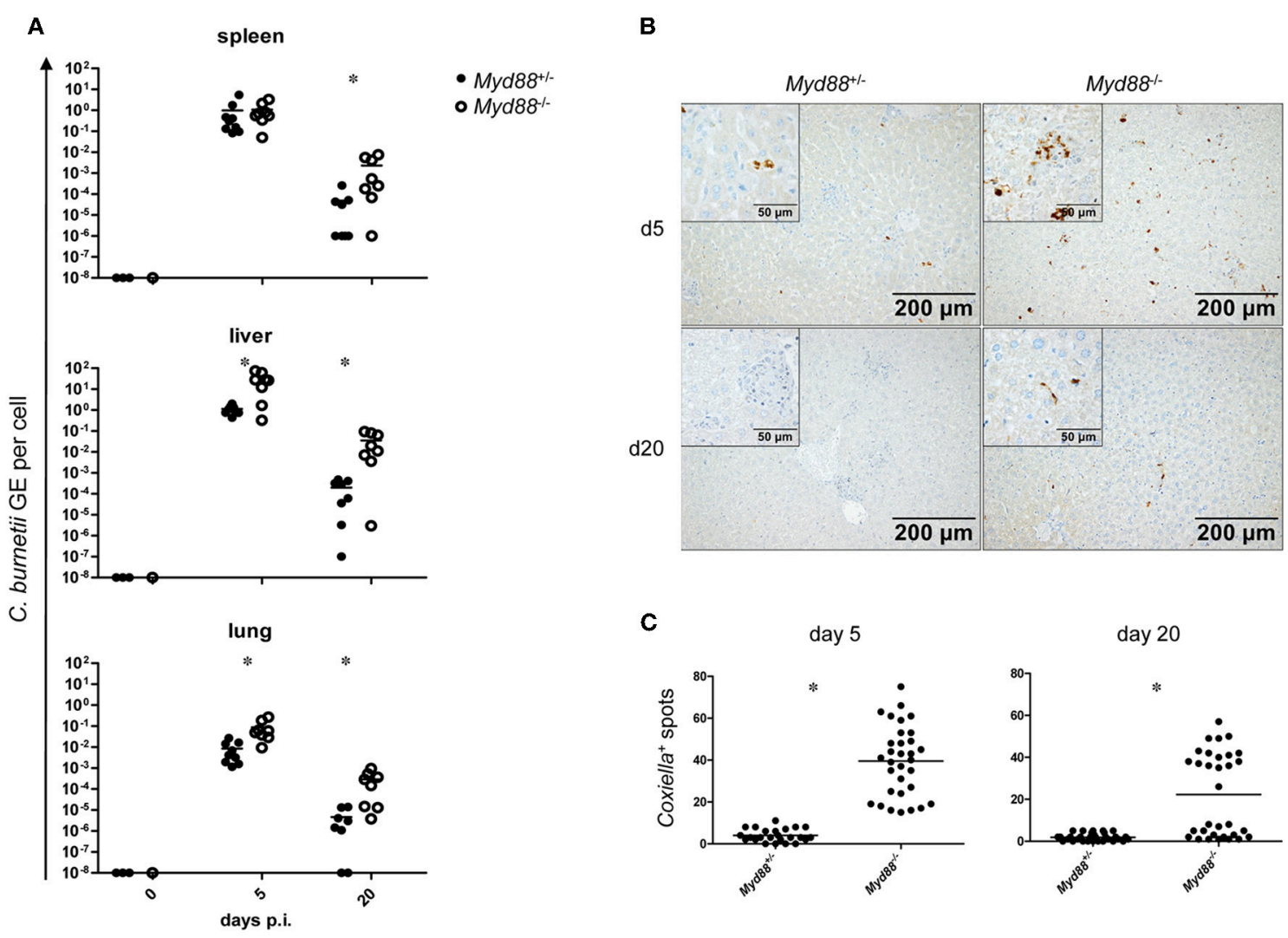

FIGURE 4 | Increased bacterial load in Myd88-/- mice after intraperitoneal infection. Mice were infected i.p. with $5 \times 10^{7}$ CFU NMII and sacrificed after 5 and 20 days. (A) bacterial burden in spleen, liver and lung was assessed by qPCR for the C. burnetii insertion sequence IS1111 and for the murine albumin gene. Shown are NMII GE per cell. Each dot in the graphs represents one single mouse; data were pooled from two independent experiment. (B) immunohistochemical staining of liver sections with anti-C. burnetii antiserum shown at 100x magnification. (Inset) enlarged view at 400x magnification. (C) quantitation of Coxiella-containing cells (dark spots) per field of view at 100x magnification was performed for eight randomly chosen positions on sections from 4 individual mice per condition. Results were analyzed using an unpaired two-tailed Mann-Whitney $U$ test.

of $\mathrm{Myd} 88^{+/-}$mice granuloma-like structures consisting of infiltrated leukocytes were easily detectable on day 5 and to a lesser extent on day 20 after infection, reflecting the massive reduction in bacterial load at the later time point in $\mathrm{Myd88^{+/- }}$ mice (Figure 4). In contrast, the granuloma size appeared smaller in $M y d 88^{-/-}$livers. Indeed, quantitative assessment of the area occupied by granulomatous inflammation showed a reduced response in the absence of MyD88 at both time points. Thus, despite a more than 1.5-log higher bacterial burden (Figure 4), $M y d 88^{-/-}$mice generated only a weak granulomatous reaction to NMII in the liver, indicating that MyD88 signaling is essential to attract leukocytes to the sites of infection. Therefore, we next turned to analyze the expression of chemokines and cytokines involved in leukocyte recruitment and in the organization of granulomatous responses.

\section{MyD88-Deficiency Thwarts Expression of Chemokines and of the IFN $\gamma$ Response in NMII-infected Mice}

The chemokine CCL2 (= MCP-1) attracts CCR2 ${ }^{+}$monocytes to sites of infection. Expression of $\mathrm{Ccl} 2$ was induced on day
5 after NMII infection and showed a clear dependence on MyD88. A similar pattern was found for the pleiotropic cytokine IL-6 and for IL-1a and IL-1b (Figure 7A). The findings of a significant reduction of CCL2 and IL- 6 in $M y d 88^{-/-}$mice in response to NMII infection are consistent with the observed reduction in leukocyte infiltration and granuloma response. IFN $\gamma$ is pivotal for the control of C. burnetii replication in vivo (3). Its expression was reduced in a $M y d 88^{-/-}$mice after infection with NMII (Figure 7B). Among the plethora of IFN $\gamma$-induced genes, iNOS stands out as an essential mediator of protection against intracellular infection in general and is required for control of C. burnetii replication in vivo in particular. MyD88-deficiency strongly impaired the induction of Nos2 expression in infected mice (Figure 7B). An even more pronounced dependence on MyD88 signaling was observed for upregulation of $G b p 1$, a member of the IFN $\gamma$-induced p65 GTPase family (Figure 7B). The chemokine CXCL10 (= IP-10) is strongly induced by IFN $\gamma$ in combination with TLR signals, and was also dependent on MyD88 signaling in NMII-infected mice (Figure 7B). In contrast to the clear MyD88-dependence of these chemokines and IFN $\gamma$ response genes, the induction of the immunoregulatory cytokine 

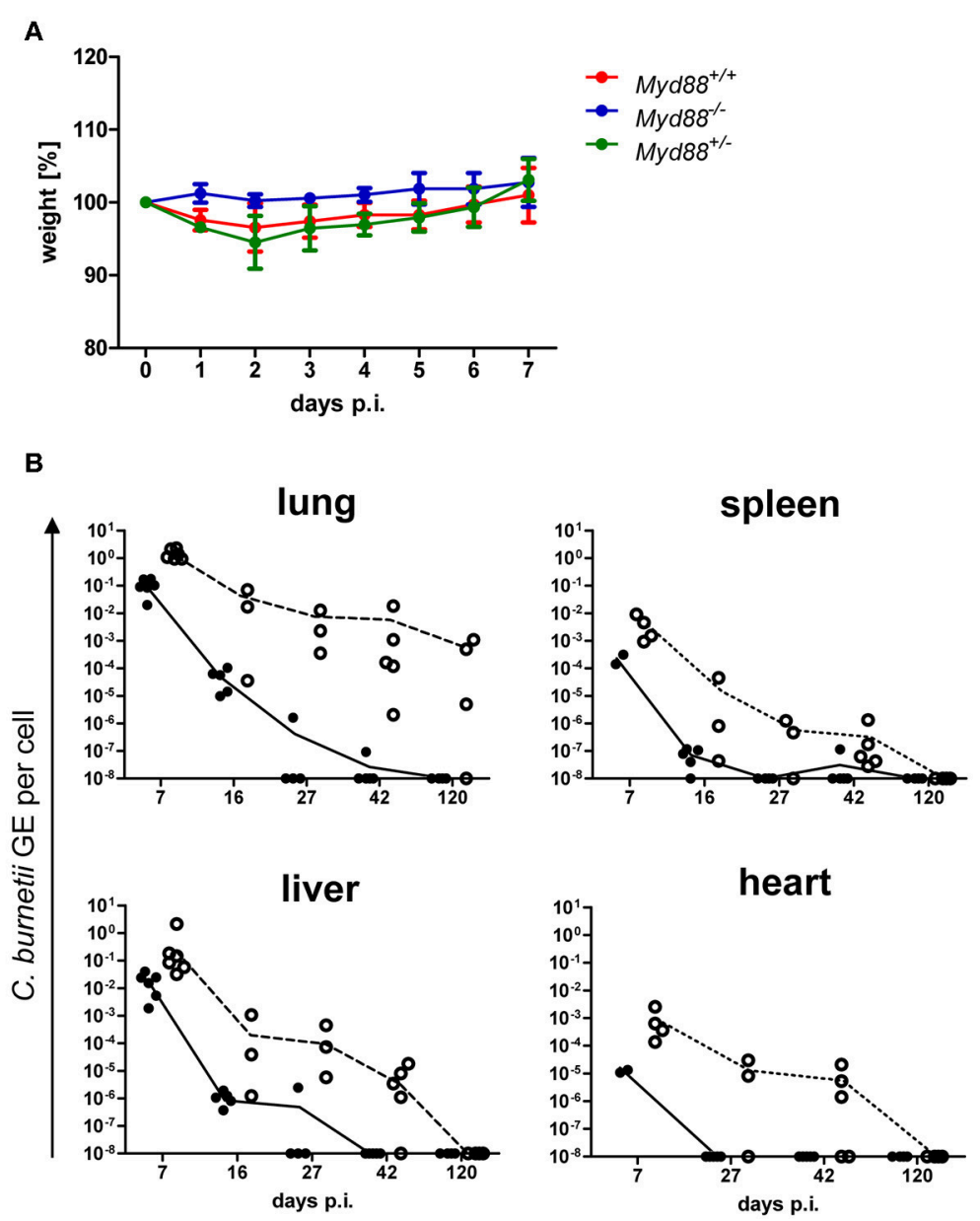

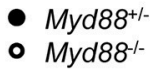

FIGURE 5 | Kinetics of $C$. burnetii persistence after intratracheal infection. Intratracheal infection of mice was performed with $10^{6}$ CFU C. burnetii NMII. (A) The weight

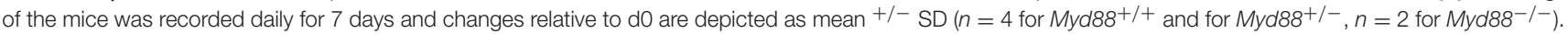
(B) mice were sacrificed on day 7, 16, 27, 42, or 120 post infection. Bacterial load in lung, spleen, liver and heart was determined via qPCR as NMII GE per cell. Every single dot in the graphs represents one single mouse.

IL-10 and of the enzyme Arginase-1 by NMII was largely independent of MyD88 (Figure 7C), which contrasts to the results obtained with BMM after infection with NMII in vitro (Figure 2). Together, MyD88 appears to be differentially required in vivo for the expression of inflammatory genes and of the IFN $\gamma$ response, but not for genes associated with alternative activation of macrophages such as Il10 and Arg1.

\section{DISCUSSION}

This manuscript demonstrates an essential role for the TLR/IL1R-associated adapter protein MyD88 in the control of $C$. burnetii replication in macrophages in vitro and for efficient local containment and elimination of the bacteria after infection in vivo. In the absence of $\mathrm{MyD} 88$, the expression and production of important inflammatory cytokines, chemokines and antimicrobial effector molecules was severely impaired. These data on host gene expression after infection provide clues about MyD88-dependent candidate mechanisms involved in immunological control of C. burnetii. Our findings are consistent with previous studies using TLR2- and MyD88-deficient mice $(12,15,27)$ and with the association of a polymorphism in human Myd88 with the development of chronic Q fever (11). Our data also show that infection of $M y d 88^{-/-}$mice with the attenuated NMII strain provides a model for prolonged and systemic infection with C. burnetii, facilitating in vivo studies of the host- $C$. burnetii interaction and the resolution of inflammation.

Since MyD88 is a central adapter protein not only of TLR signaling, but also of the receptors for IL-1, IL-18, and IL-33, the impaired control of NMII in $M y d 88^{-/-}$mice may be due to the combined lack of pathogen sensing through TLR and signaling through IL-1 family cytokines. While the role of IL-18 and IL33 in C. burnetii infection has not been investigated yet, mice deficient in the IL-1 receptor had a transiently increased bacterial burden in the lung, but not in spleen, after intranasal infection (28), suggesting that IL-1R signaling accounts for only a minor part of MyD88's protective function. The strongly decreased 
A

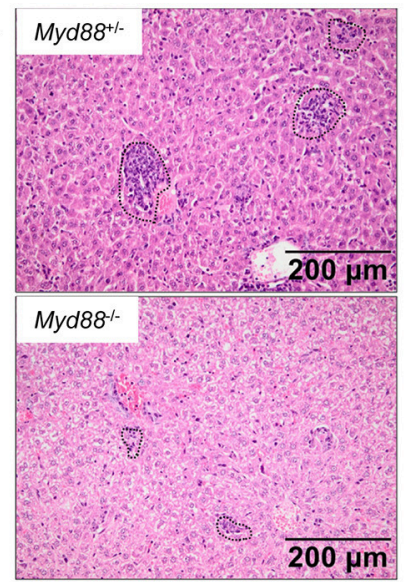

B

day 5

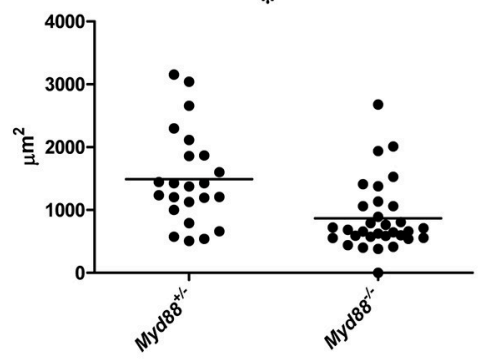

day 20

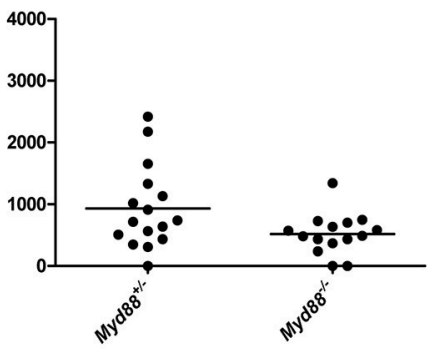

FIGURE 6 | MyD88-dependent granulomatous inflammation in livers of C. burnetii-infected mice. Myd88 ${ }^{+/-}$and Myd88 $^{-/-}$mice were infected i.p. with $5 \times 10^{7}$ CFU NMII. H\&E stained liver sections were analyzed for leukocyte infiltrates by histopathology (magnification 100x). (A) Granuloma-like structures were marked (indicated by dashed lines in photomicrographs, shown are examples from day 5 post infection) and the total area of all marked granulomas per field of view was measured (ZEN software, Zeiss). Eight fields of view were analyzed per individual mouse (B). Results were analyzed using an unpaired two-tailed Mann-Whitney $U$ test. (d5: $n=3$ for Myd88+/-, $n=4$ for Myd88 ${ }^{-/-}$; d20: $n=2$ for both genotypes).

A

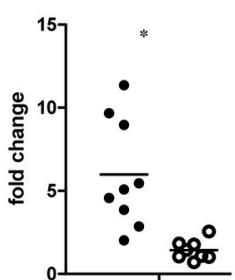

B
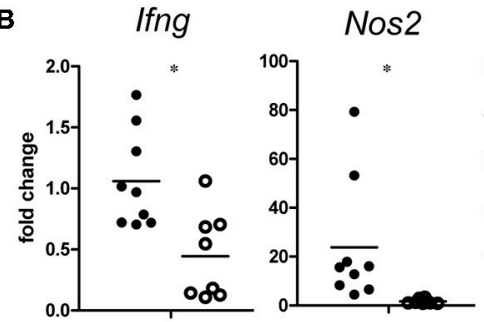

C

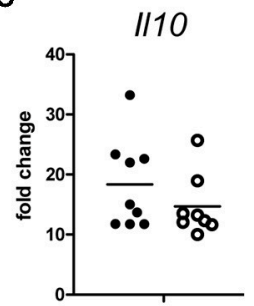

Ccl2
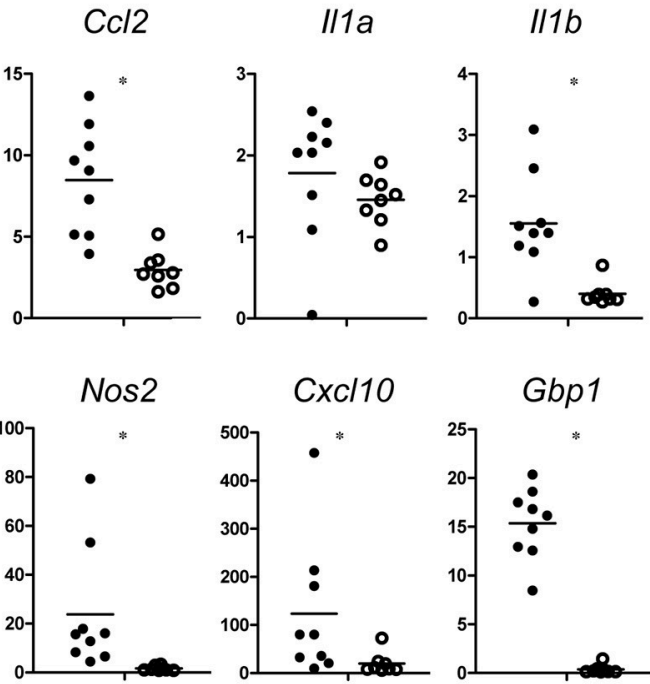

- $M y d 88^{+1-}$

- Myd88-1-

FIGURE 7 | MyD88-dependence of gene expression changes in C. burnetii-infected mice. On day 5 after i.p. infection with NMII (5 × $10^{7}$ CFU), RNA from spleen was harvested and analyzed by qRT-PCR. Fold changes were calibrated to non-infected Myd88 $+/-$ mice. Each dot represents one mouse. Data were pooled from two independent experiments. (A) expression of the pro-inflammatory cytokines $/ / 6$, CCl2, /11a, and //1b. (B) expression of Ifng and its target genes Nos2, Cxc/10, and Gbp1. (C) expression of //10 and Arg1. Results were analyzed using an unpaired two-tailed Mann-Whitney U-test. 
induction of IL-1 expression observed by us in the absence of MyD88 (Figure 7) is consistent with the notion that its function as adapter of TLRs upstream of IL-1 and other TLR-induced cytokines is critical to initiate protective immunity.

An involvement of TLR2 in innate immune sensing of $C$. burnetii was already shown using macrophages in vitro (12). However, Tlr $2^{-/-}$mice were able to clear C. burnetii after intraperitoneal infection comparable to wild-type mice (27). More recently, Ramstead et al. demonstrated a requirement for TLR2 in protection against C. burnetii infection in mice after intratracheal, but not intraperitoneal infection (15). The same study also demonstrated that MyD88-deficient mice have a more dramatic increase in bacterial burden than Tlr2 knockouts after intratracheal infection with virulent $C$. burnetii Nine Mile phase I (15). While our results corroborate the importance of intact MyD88-dependent signaling reported after infection with C. burnetii Nine Mile phase I using the attenuated NMII strain, it is important to point out the additional differences between both studies: first, we infected $M y d 88^{-/-}$mice by both the intraperitoneal and the intratracheal route, showing that a robust increase in bacterial load was observed independent of the route of infection; second, we performed a much longer kinetic sampling that showed the prolonged presence of NMII in the lung and other organs; third, we included a comprehensive analysis of host gene expression changes after infection.

Our results indicate that deficiency in MyD88 impairs control of NMII at the level of innate as well as adaptive immunity. First, the lack of robust production of cytokines like TNF by macrophages infected with NMII is sufficient to explain the unrestricted replication in vitro (Figure 1), as recently demonstrated in TNF-deficient macrophages (14), and likely contributes to the increased bacterial burden during the early phase of infection in vivo. Impaired expression of TNF and of chemokines like CCL2 in MyD88-deficient tissues allows only a weak granulomatous response (Figure 6) with reduced production of IFN $\gamma$, likely by $\mathrm{T}$ cells, and in consequence suboptimal expression of anti-microbial effectors (Figure 7). Among these, iNOS is established as important contributor of protection against C. burnetii (4). Expression of Gbp1, a member of the $65 \mathrm{kDa}$ family of IFN $\gamma$-induced GTPases, was abrogated in NMII-infected Myd88 ${ }^{-/-}$mice (Figure 7). Gbp1 is recruited to the phagosomes of macrophages infected with mycobacteria and is required for control of mycobacterial replication in mice (29). Whether Gbp1, and other members of this family, is essential for inhibition of replication of C. burnetii will be important to test in future experiments. Of note, not all host response genes to NMII infection were MyD88-dependent (Figures 2, 7). The induction of iNOS in macrophages in vitro, and the expression of Arginase1 and of IL-10 in vivo, was not altered in MyD88-deficient cells/mice after infection with NMII. These findings demonstrate that most likely additional pattern recognition receptors or adapter protein pathways are triggered by C. burnetii, which may differ between infected cell types in vitro and in vivo.

Regardless of the mechanism by which MyD88 counteracts the spread and replication of NMII, the relevance of the findings in the mouse model for the course of human $\mathrm{Q}$ fever disease is indicated by a recent investigation of genetic variation in pattern recognition receptors and adapter proteins in patients with chronic Q fever and controls with previous exposure to C. burnetii, which identified a SNP in the Myd88 promoter (938C $>$ A) with susceptibility to develop chronic Q fever (11). The robust differences in bacterial loads observed here in $M y d 88^{-/-}$ mice, indicate that infection with NMII can be successfully employed to model chronicity of Q fever. In fact, the increase in bacterial burden after intratracheal infection was considerably more pronounced than that observed in the only described model for chronic $Q$ fever to date, the macIL-10tg mouse line overexpressing IL-10 from macrophages, after infection with the virulent C. burnetii Nine Mile phase I strain (10). In this context, it is also important to note that C. burnetii infection models in macIL-10tg and $M y d 88^{-/-}$mice lead to prolonged bacterial persistence in the organs through different mechanism: first, deficient sensing of Coxiella-derived PAMPs

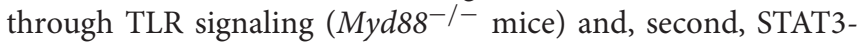
dependent deactivation of TLR-induced inflammatory responses and reprogramming of macrophages (in macIL-10tg mice). Importantly, both models represent different pathophysiological mechanisms apparently operating in patients with chronic Q fever $(7,11)$. The finding that the attenuated $C$. burnetii strain used by us causes persistent infection in $M y d 88^{-/-}$mice is in line with the recent appreciation of the NMII strain as causing lethal infection in immunocompromised SCID mice, showing that virulence of $C$. burnetii cannot be reduced to phase I LPS alone $(22,23)$. In contrast to SCID mice, that completely lack adaptive immunity, the $M y d 88^{-/-}$mouse model has the advantage of a less severely compromised immune system and therefore much better mimics the situation in humans.

\section{MATERIALS AND METHODS}

\section{Generation of Bone Marrow Derived Macrophages}

All mice utilized in this study were at least 6 weeks of age. C57BL/6 wildtype mice were obtained from Charles River Breeding Laboratories (Sulzfeld, Germany). The MyD88deficient mice were generated by Dr. S. Akira (30), backcrossed to $\mathrm{C} 57 \mathrm{BL} / 6$, and bred at the Präklinische Experimentelle Tierzentrum of the University Hospital Erlangen.

Primary bone marrow derived macrophages (BMM) from femurs and tibiae were propagated by culture in petri dishes for $7 \mathrm{~d}$ in complete Dulbecco's modified Eagle medium (DMEM) (Life Technologies) containing 10\% fetal bovine serum (FBS) (Biochrome), antibiotics, and $50 \mu \mathrm{M} \beta$-mercaptoethanol (complete DMEM [cDMEM]) plus 10\% L929 cell-conditioned medium as a source of macrophage colony-stimulating factor (M-CSF) at $37^{\circ} \mathrm{C}, 10 \% \mathrm{CO} 2$ and $21 \% \mathrm{O} 2$. Adherent macrophages were harvested by Accutase (Sigma, Deisenhofen, Germany) treatment, washed, and counted.

\section{Culture of Coxiella Burnetii}

An isolate of the C. burnetii Nine Mile phase II strain clone 4 (NMII, RSA493) was generously provided by Matteo Bonazzi (Institut de Recherche en Infectiologie de Montpellier, Montpellier, France). One aliquot of purified C. burnetii NMII 
was propagated in a $75 \mathrm{~cm}^{2}$ tissue culture flask containing $30 \mathrm{ml}$ acidified citrate cysteine medium (ACCM-2, 4700-003, Sunrise Science Products, San Diego, CA, USA) in an atmosphere of $37^{\circ} \mathrm{C}, 5 \% \mathrm{CO}_{2}$ and $2.5 \% \mathrm{O}_{2}$ (31). Following 4 days of culture, C. burnetii were transferred overnight to room temperature and ambient atmosphere. Subsequently, bacteria were pelleted for $30 \mathrm{~min}$ at $4500 \mathrm{xg}$, resuspended in $1 \mathrm{ml}$ phosphate buffered saline (PBS) and quantified by optical density at $\mathrm{OD}_{600}$, where an $\mathrm{OD}_{600}$ of 1 equals $\sim 1 \times 10^{9} \mathrm{C}$. burnetii per $\mathrm{ml}$, as determined by serial dilution, plating on ACCM-D agarose and counting of CFU after 9 days of culture.

\section{In vitro Infection of BMM}

$2 \mathrm{~h}$ prior infection, BMM were harvested, seeded and cultured at $37^{\circ} \mathrm{C}, 5 \% \mathrm{CO}_{2}$ in RPMI complete medium containing $10 \%$ fetal calf serum (FCS), 1\% HEPES and $0.5 \% \beta$-mercaptoethanol (RPMI-CM). For microscopic analysis, $0.5 \times 106 \mathrm{BMM}$ were seeded on $10 \mathrm{~mm}$ coverslips in 24-well dishes, whereas 12 -well dishes and a density of $1.0 \times 10^{6}$ cells/well were used for qPCR and cytokine ELISA studies. The cells were washed with PBS and infected with C. burnetii at MOI 10. Following infection, cells were centrifuged for $5 \mathrm{~min}$ at $250 \mathrm{x}$ g and incubated at $37^{\circ} \mathrm{C}$ and $5 \% \mathrm{CO}_{2}$. After $4 \mathrm{~h}$, cells were washed with PBS to remove extracellular C. burnetii and supplied with RPMI-CM.

\section{Quantification of Bacterial Load}

We defined the ratio of $C$. burnetii genomic copies to BMM genomic copies as bacterial load per cell. C. burnetii genomes were quantified from isopropanol-precipitated DNA samples performing quantitative real-time PCR (qPCR) with a primer set specific for $\operatorname{dot} A$ gene as described elsewhere (32). BMM genomic copies were quantified from the same sample using a primer set specific for murine albumin gene (exon 7): forward, 5'-GGCAACAGACCTGACCAAAG-3' and reverse, 5'-CAGCAACCAAGGAGAGCTTG-3'. Isopropanolpurified DNA from $10^{8} \mathrm{BMM}$ was diluted 10 -fold down to 10 and served as a template to generate standard curves. qPCR was carried out in 384-well optical plates on an ABI Prism 7900HT sequence-detection system (life technologies) with $5 \times$ EvaGreen QPCR MixII-ROX (Bio\&Sell, Fürth, Germany), a $0.1 \mu \mathrm{M}$ final concentration of each primer and $2 \mu$ isolated DNA (pediluted 1:20 in ultrapure $\mathrm{H}_{2} \mathrm{O}$ ) as template in a final volume of $10 \mu \mathrm{l}$ per reaction.

\section{Cytokine ELISA}

Cytokine production of C. burnetii infected BMM was assessed from supernatants (SN). SNs were collected, centrifuged at 500 $\times \mathrm{g}, 4^{\circ} \mathrm{C}$ for $10 \mathrm{~min}$, transferred to a new $1.5 \mathrm{ml}$ reaction tube and stored at $-80^{\circ} \mathrm{C}$ until they were analyzed. Enzymelinked immunosorbent assay (ELISA) kits (BDTM, Heidelberg, Germany) specific for the indicated cytokine were applied according to the manufacturer's protocol.

\section{Immunofluorescence}

For indirect immunofluorescence microscopy analyses, $C$. burnetii infected BMM were cultured on $10 \mathrm{~mm}$ coverslips in 24well dishes. At indicated points of time, cells were washed three times with equilibrated PBS, fixed for 15 min with equilibrated $4 \%$ paraformaldehyde (PFA) and permeabilized with ice-cold methanol for $1 \mathrm{~min}$. Cells were quenched and blocked with $50 \mathrm{mM} \mathrm{NH}_{4} \mathrm{Cl}$ in PBS/ 5\% goat serum (GS) for $60 \mathrm{~min}$ at room temperature. Incubation with primary antibody dilution in PBS/ 5\% GS was conducted at room temperature for $60 \mathrm{~min}$. Subsequently, cells were washed three times with PBS and further incubated with secondary antibodies in PBS/ 5\% GS for $30 \mathrm{~min}$ at room temperature. After final $3 \mathrm{x}$ washing with PBS, coverslips were mounted using ProLong Diamond containing DAPI. For visualization, a Carl Zeiss LSM 700 Laser Scan Confocal Microscope and the ZEN2009 software (Jena, Germany) were used.

In this study, we used primary antibodies directed against $C$. burnetii and LAMP-1 (Developmental Studies Hybridoma Bank, Iowa, IA, USA). Secondary antibodies were Alexa Fluor labeled (Alexa Fluor 488/ green, 594/ red) and purchased from Dianova, Hamburg, Germany.

\section{In vivo Infection Experiments}

All mouse experiments were approved by the regional government (Regierung von Unterfranken, animal protocol 54-2532.1-44/13). Six to twelve week old $M y d 88^{+/+}, M y d 88^{+/-}$ and $M y d 88^{-/-}$mice (on C57BL/6 background) were bred in the Präklinische Experimentelle Tierzentrum (University Hospital Erlangen) and housed in ventilated ISOcages under S2 conditions. Sex matched groups of mice were infected with C. burnetii either intraperitoneally $\left(5 \times 10^{7} \mathrm{CFU} / 200 \mu \mathrm{l}\right.$ $\mathrm{PBS} /$ mouse) or intratracheally $\left(10^{6} \mathrm{CFU} / 50 \mu \mathrm{l} \mathrm{PBS} / \mathrm{mouse}\right)$. Intratracheal infection was performed by direct injection of bacterial suspension into the trachea of the mice. To do this, mice were anesthetized with isofluorane, placed in dorsal position and the trachea was accessed after incision of the skin. Prior to surgery buprenorphine $(0.1 \mathrm{mg} / \mathrm{kg})$ was injected intraperitoneally for analgesia. The physical condition of the mice was monitored regularly. At the indicated time points, mice were humanely killed and organ tissue ( $\sim 20-50 \mathrm{mg}$ ) of spleen, liver, lung, heart was collected. Tissue samples were preserved in PeqDirectLysis ${ }^{\circledR}$ buffer (Peqlab) + Proteinase K (Roche) for DNA preparation or RNAlater ${ }^{\circledR}$ (Qiagen) for subsequent RNA isolation. For histopathology, organ pieces were fixed in PFA 4\%.

\section{DNA Isolation and Quantification of C. burnetii Burden Via qPCR}

Organ tissue was lysed in PeqDirectLysis ${ }^{\circledR}$ buffer + Proteinase $\mathrm{K}$ at $56^{\circ} \mathrm{C}$ under shaking overnight. Then, Proteinase $\mathrm{K}$ was inactivated at $85^{\circ} \mathrm{C}$. DNA from organ tissue then directly was used for qPCR. To amplify C. burnetii DNA, a TaqMan-based quantitative PCR for the insertion sequence IS1111 was performed using 5'-CATCGTTCCCGGCAGTT$3^{\prime}$ as forward, 5'-TAATCCCCAACAACACCTCCTTA-3' as reverse primer, and the internal fluorogenic probe 6FAMCGAATGTTGTCGAGGGACCAACCCAATAAA-BBQ

(TibMolbiol, Berlin, Germany). To quantitate C. burnetii genome equivalents, a standard curve using titrated DNA prepared from a defined number of in vitro cultured C. burnetii NMII was included in each experiment. A separate qPCR run 
was performed in parallel for mouse albumin gene copies, using titrated DNA prepared from mouse splenocytes for standard curve generation. C. burnetii burden per cell was calculated by normalizing bacterial genome copy numbers to albumin copy numbers.

\section{RNA Isolation and Gene Expression Analysis by qRT-PCR}

RNA from organ tissue (stored in RNAlater ${ }^{\circledR}$ stabilizing reagent until further processing) was isolated using peqGold TriFastTM (Peqlab) and cDNA was synthesized using High Capacity cDNA Reverse Transcription Kit (Applied Biosystems). Primers and probes were chosen from the Universal ProbeLibrary (Roche). The fold change in gene expression was determined by the $\Delta \triangle \mathrm{CT}$ method, using HPRT as housekeeping gene for calculation of $\Delta \mathrm{CT}$ values and samples of naïve $M y d 88^{+/-}$mice as calibrators.

\section{Processing of Organ Tissue for Histopathological Analysis}

After fixation of organ pieces for $24 \mathrm{~h}$ in PFA $4 \%$ at $4{ }^{\circ} \mathrm{C}$ tissue was washed with PBS and subsequently embedded in paraffin. Five micrometers of paraffin-embedded tissue slides were transferred to Star Frost ${ }^{\circledR}$ microscope slides for staining of the tissue.

\section{H\&E Staining of Organ Tissue}

Paraffin-embedded tissue sections were stained with Hematoxilin and Eosin (H\&E) to visualize alterations in tissue structure and tissue-infiltrating leukocytes. Leukocyte infiltrates were marked by a blinded investigator and the area of each granuloma-like structure was calculated using ZEN software (Zeiss).

\section{REFERENCES}

1. Fenollar F, Fournier PE, Carrieri MP, Habib G, Messana T, Raoult D. Risks factors and prevention of Q fever endocarditis. Clin Infect Dis. (2001) 33:3126. doi: 10.1086/321889

2. Kampschreur LM, Dekker S, Hagenaars JC, Lestrade PJ, Renders NHM, de Jager-Leclercq MGL, et al. Identification of risk factors for chronic Q fever, The Netherlands. Emerg Infect Dis. (2012) 18:563-70. doi: 10.3201/eid1804.111478

3. Andoh M, Zhang G, Russell-Lodrigue KE, Shive HR, Weeks BR, Samuel JE. $T$ cells are essential for bacterial clearance, and gamma interferon, tumor necrosis factor alpha, and B cells are crucial for disease development in Coxiella burnetii infection in mice. Infect Immun. (2007) 75:3245-55. doi: 10.1128/IAI.01767-06

4. Brennan RE, Russell K, Zhang G, Samuel JE. Both inducible nitric oxide synthase and NADPH oxidase contribute to the control of virulent phase I Coxiella burnetii infections. Infect Immun. (2004) 72:6666-75. doi: 10.1128/IAI.72.11.6666-6675.2004

5. Schoffelen T, Textoris J, Bleeker-Rovers CP, Ben Amara A, van der Meer JW, Netea MG. Intact interferon-gamma response against Coxiella burnetii by peripheral blood mononuclear cells in chronic Q fever. Clin Microbiol Infect. (2017) 23:209 e209-209 e215. doi: 10.1016/j.cmi.2016.11.008

6. Lang R. Tuning of macrophage responses by Stat3-inducing cytokines: molecular mechanisms and consequences in infection. Immunobiology (2005) 210:63-76. doi: 10.1016/j.imbio.2005.05.001

7. Honstettre A, Imbert G, Ghigo E, Gouriet F, Capo C, Raoult D, et al. Dysregulation of cytokines in acute $\mathrm{Q}$ fever: role of interleukin-10 and tumor necrosis factor in chronic evolution of Q fever. J Infect Dis. (2003) 187:956-62. doi: $10.1086 / 368129$

\section{Immunohistochemistry Staining of Organ Tissue}

C. burnetii was detected using the polyclonal $\alpha-C$. burnetii antiserum described above. Detection of bound antibodies was performed with biotinylated secondary goat ant-rabbit antibody and $\mathrm{ABC}-\mathrm{Kit}$ using the peroxidase substrate method DABImmPact (all from Vector Laboratories, Burlingame, CA, USA). Quantification of C. burnetii positive spots in eight randomly chosen fields of view in the tissue sections was done at 100x magnification using ZEN software (Zeiss).

\section{Statistical Analysis}

Statistical analysis was performed as described individually for each graph in the figure legends using either GraphPad Prism (version 5) or SigmaPlot. $P<0.05$ were statistically significant and are indicated by an asterisk $(*)$.

\section{AUTHOR CONTRIBUTIONS}

LK, IH, CD, JS-L, and BB performed experiments. LK, IH, CD, JS-L, AL, and RL designed and analyzed experiments. RL wrote the manuscript with input from LK, IH, CD, JS-L, and AL.

\section{ACKNOWLEDGMENTS}

Support by the Deutsche Forschungsgemeinschaft SFB 1181, $\mathrm{TP} A 06$, to $\mathrm{RL}$ and $\mathrm{AL}$ is gratefully acknowledged. The technical assistance of K. Jozefowski, M. Oehlke, W. Espach and S. Soellner, and the help of M. Kandler in granuloma quantitation, is appreciated.

8. Ghigo E, Honstettre A, Capo C, Gorvel JP, Raoult D, Mege JL. Link between impaired maturation of phagosomes and defective Coxiella burnetii killing in patients with chronic Q fever. J Infect Dis. (2004) 190:1767-72. doi: 10.1086/425041

9. Lang R, Rutschman RL, Greaves DR, Murray PJ. Autocrine deactivation of macrophages in transgenic mice constitutively overexpressing IL-10 under control of the human CD68 promoter. J Immunol. (2002) 168:3402-11. doi: 10.4049/jimmunol.168.7.3402

10. Meghari S, Bechah Y, Capo C, Lepidi H, Raoult D, Murray P, et al. Persistent Coxiella burnetii infection in mice overexpressing IL-10: an efficient model for chronic Q fever pathogenesis. PLoS Pathog. (2008) 4:e23. doi: 10.1371/journal.ppat.0040023

11. Schoffelen T, Ammerdorffer A, Hagenaars JC, Bleeker-Rovers CP, Wegdam-Blans MC, Wever PC, et al. Genetic variation in pattern recognition receptors and adaptor proteins associated with development of chronic Q fever. J Infect Dis. (2015) 212:818-29. doi: 10.1093/infdis /jiv113

12. Zamboni DS, Campos MA, Torrecilhas AC, Kiss K, Samuel JE, Golenbock DT, et al. (2004). Stimulation of toll-like receptor 2 by Coxiella burnetii is required for macrophage production of pro-inflammatory cytokines and resistance to infection. J Biol Chem. 279:54405-15. doi: 10.1074/jbc.M410340200

13. Ammerdorffer A, Schoffelen T, Gresnigt MS, Oosting M, den Brok MH, Abdollahi-Roodsaz S, et al. Recognition of Coxiella burnetii by toll-like receptors and nucleotide-binding oligomerization domain-like receptors. $J$ Infect Dis. (2015) 211:978-87. doi: 10.1093/infdis/jiu526

14. Bradley WP, Boyer MA, Nguyen HT, Birdwell LD, Yu J, Ribeiro J., et al. Primary role for toll-like receptor-driven tumor necrosis factor rather than cytosolic immune detection in restricting coxiella burnetii phase II 
replication within mouse macrophages Infect Immun. (2016) 84:998-1015. doi: 10.1128/IAI.01536-15

15. Ramstead AG, Robison A, Blackwell A, Jerome M, Freedman B, Lubick $\mathrm{K}$, et al. Roles of toll-like receptor 2 (TLR2), TLR4, and MyD88 during pulmonary coxiella burnetii infection. Infect Immun. (2016) 84:940-9. doi: 10.1128/IAI.00898-15

16. Eldin C, Melenotte C, Mediannikov O, Ghigo E, Million M, Edouard S, et al. From Q fever to Coxiella burnetii infection: a paradigm change. Clin Microbiol Rev. (2017) 30:115-90. doi: 10.1128/CMR.00045-16

17. Hoover TA, Culp DW, Vodkin MH, Williams JC, Thompson HA. Chromosomal DNA deletions explain phenotypic characteristics of two antigenic variants, phase II and RSA 514 (crazy), of the Coxiella burnetii nine mile strain. Infect Immun. (2002) 70:6726-33. doi: 10.1128/IAI.70.12.6726-2733.2002

18. Moos A, Hackstadt T. Comparative virulence of intra- and interstrain lipopolysaccharide variants of Coxiella burnetii in the guinea pig model. Infect Immun. (1987) 55:1144-50.

19. Ormsbee R, Peacock M, Gerloff R, Tallent G, Wike D. Limits of rickettsial infectivity. Infect Immun. (1978) 19:239-45.

20. Graham JG, MacDonald LJ, Hussain SK, Sharma UM, Kurten RC, Voth DE. Virulent Coxiella burnetii pathotypes productively infect primary human alveolar macrophages. Cell Microbiol. (2013) 15:1012-25. doi: $10.1111 / \mathrm{cmi} .12096$

21. Howe D, Shannon JG, Winfree S, Dorward DW, Heinzen RA. (2010). Coxiella burnetii phase I and II variants replicate with similar kinetics in degradative phagolysosome-like compartments of human macrophages. Infect Immun. 78:3465-74. doi: 10.1128/IAI.0 0406-10

22. Islam A, Lockhart M, Stenos J, Graves S. The attenuated nine mile phase II clone 4/RSA439 strain of Coxiella burnetii is highly virulent for severe combined immunodeficient (SCID) mice. Am J Tropical Med Hyg. (2013) 89:800-3. doi: 10.4269/ajtmh.12-0653

23. van Schaik EJ, Case ED, Martinez E, Bonazzi M, Samuel J. The SCID mouse model for identifying virulence determinants in Coxiella burnetii. Front Cell Infect Microbiol. (2017) 7:25. doi: 10.3389/fcimb.2017. 00025

24. Howe D, Barrows LF, Lindstrom NM, Heinzen R. Nitric oxide inhibits Coxiella burnetii replication and parasitophorous vacuole maturation. Infect Immun. (2002) 70:5140-7. doi: 10.1128/IAI.70.9.5140-5147.2002
25. Calverley M, Erickson S, Read AJ, Harmsen AG. Resident alveolar macrophages are susceptible to and permissive of Coxiella burnetii infection. PLoS ONE (2012) 7:e51941. doi: 10.1371/journal.pone.0051941

26. Fernandes TD, Cunha LD, Ribeiro JM, Massis L, Lima-Junior DS, Newton $\mathrm{JH}$, et al. Murine alveolar macrophages are highly susceptible to replication of coxiella burnetii phase II in vitro. Infect Immun. (2016) 84:2439-48. doi: 10.1128/IAI.00411-16

27. Meghari S, Honstettre A, Lepidi H, Ryffel B, Raoult D, Mege J, et al. TLR2 is necessary to inflammatory response in Coxiella burnetii infection. Ann N Y Acad Sci. (2005) 1063:161-6. doi: 10.1196/annals.1355.025

28. Elliott A, Schoenlaub L, Freches D, Mitchell W, Zhang G. Neutrophils play an important role in protective immunity against Coxiella burnetii infection. Infect Immun. (2015) 83:3104-13. doi: 10.1128/IAI.00042-15

29. Kim BH, Shenoy AR, Kumar P, Das R, Tiwari S, MacMicking JD. A family of IFN-gamma-inducible $65-\mathrm{kD}$ GTPases protects against bacterial infection. Science (2011) 332:717-21. doi: 10.1126/science.1201711

30. Adachi O, Kawai T, Takeda K, Matsumoto M, Tsutsui H, Sakagami $\mathrm{M}$, et al. Targeted disruption of the MyD88 gene results in loss of IL-1- and IL-18-mediated function. Immunity (1998) 9:143-50. doi: 10.1016/S1074-7613(00)80596-8

31. Omsland A, Cockrell DC, Howe D, Fischer ER, Virtaneva K, Sturdevant DE, et al. (2009). Host cell-free growth of the Q fever bacterium Coxiella burnetii. Proc Natl Acad Sci USA. 106:4430-4. doi: 10.1073/pnas.0812074106

32. Schulze-Luehrmann J, Eckart RA, Ölke M, Saftig P, Liebler-Tenorio E, Lührmann A. LAMP proteins account for the maturation delay during the establishment of the Coxiella burnetii-containing vacuole. Cell Microbiol. (2016) 18:181-94. doi: 10.1111/cmi.12494

Conflict of Interest Statement: The authors declare that the research was conducted in the absence of any commercial or financial relationships that could be construed as a potential conflict of interest.

Copyright (C) 2019 Kohl, Hayek, Daniel, Schulze-Lührmann, Bodendorfer, Lührmann and Lang. This is an open-access article distributed under the terms of the Creative Commons Attribution License (CC BY). The use, distribution or reproduction in other forums is permitted, provided the original author(s) and the copyright owner(s) are credited and that the original publication in this journal is cited, in accordance with accepted academic practice. No use, distribution or reproduction is permitted which does not comply with these terms. 